\title{
The 'tumble' departure mode in weightshift-controlled microlight aircraft
}

\author{
G Gratton $^{1}$ and S Newman ${ }^{2 *}$ \\ ${ }^{1}$ British Microlight Aircraft Association, Banbury, Oxfordshire, UK \\ ${ }^{2}$ Department of Aerospace Engineering, University of Southampton, UK
}

\begin{abstract}
The cost of private or recreational flying is high for most conventional aircraft types. During the last 25 years, however, an alternative has become available in the form of the microlight aircraft. It has a relatively low cost of ownership and has opened up flying to a greater audience.

However, there have been a number of accidents, usually fatal, to this class of aircraft, which could not be explained through any conventional understanding. The reason for these accidents, which involve a departure from controlled flight followed by aircraft structural failure (generally including mechanical failure of the basebar, wingtips and leading edge), has become known as the 'tumble', owing to the basic motion of the aircraft. This paper analyses the tumble mode, from its initiation through to the steady rotation condition. History has shown that the tumble mode has always resulted in the destruction of an aircraft. In consequence, it is the authors' opinion that consideration of the tumble during the approval of new designs should concentrate upon avoidance, since there is no identifiable recovery mechanism from the established mode without the use of an external safety device.

A programme of research into this phenomenon was initiated in 1997. The peculiar nature of the tumble motion has required the aerodynamic modelling to address the effects of a high pitch rate, which has led to the introduction of unsteady aerodynamic effects.

This paper sets out to describe and explain the mode, leading to a model that might reasonably be developed to produce relatively tumble resistant aircraft. Finally, the methods of possible entry are explained; it is intended that this information may be used in pilot training to ensure the avoidance of the tumble instability.
\end{abstract}

Keywords: tumble mode, Microlight aircraft, structural failure, weightshift control

\section{NOTATION}

\section{AoA angle of attack}

BHPA British Hang-Gliding and Paragliding

BMAA

CAVOK

$C_{\mathrm{M}}$

$\mathrm{CG}$

$D$

$D_{\mathrm{T}}$ Association

$D_{\mathrm{W}}$

IAS

IMC

$K_{\mathrm{DT}}$

$L$

$L_{\mathrm{CGW}}$ visibility greater than $10 \mathrm{~km}$, no cumulonimbus, no cloud below $5000 \mathrm{ft}$ or highest minimum safety altitude (if greater), no weather significant to aviation pitching moment centre of gravity (centre of mass) drag trike drag
$M$

MCP

MTOW

OAT

PLF

QFE
The MS was received on 19 April 2002 and was accepted after revision for publication on 13 May 2003.

* Corresponding author: Department of Aerospace Engineering, University of Southampton, Highfield, Southampton SO17 1BJ, UK. wing drag indicated air speed instrument meteorological conditions trike drag coefficient $=D / V^{2}$

lift distance of the wing $\mathrm{CG}$ behind the hangpoint (along the wing chord line) wing aerodynamic pitching moment maximum continuous power maximum authorized take-off weight outside air temperature power required to maintain level flight altimeter setting giving an indication of zero height on the ground at a destination aerodrome; given in $\mathrm{hPa}$ (heptopascals), IAS sea-level value being 1013.25 time thrust (due to powerplant) aircraft translational velocity 


\begin{tabular}{|c|c|}
\hline$V_{\mathrm{DF}}$ & $\begin{array}{l}\text { flight test air speed limit (velocity design } \\
\text { flight test) }\end{array}$ \\
\hline$V_{\mathrm{NE}}$ & $\begin{array}{l}\text { normal operational air speed limit } \\
\text { (velocity never exceeded) }\end{array}$ \\
\hline$V_{\mathrm{SO}}$ & $\begin{array}{l}\text { stalling speed at MTOW in the landing } \\
\text { configuration }\end{array}$ \\
\hline VMC & visual meteorological conditions \\
\hline$W$ & weight \\
\hline$W_{\mathrm{T}}$ & weight of trike \\
\hline$W_{\mathrm{W}}$ & weight of wing \\
\hline$W_{\mathrm{X}}$ & weather \\
\hline$x$ & $\begin{array}{l}\text { radial distance of the aerofoil about the } \\
\text { centre of rotation during tumble }\end{array}$ \\
\hline$X_{\mathrm{CGT}}$ & $\begin{array}{l}\text { perpendicular distance of the trike } \mathrm{CG} \\
\text { forwards of the monopole }\end{array}$ \\
\hline$y$ & $\begin{array}{l}\text { chordwise distance of an aerofoil section } \\
\text { forward from the tangent from the chord }\end{array}$ \\
\hline $\mathrm{CO}$ & $\begin{array}{l}\text { line that intercepts the centre of rotation } \\
\text { distance below the hangpoint of the trike } \\
\text { CG (in the axis parallel to the monopole) }\end{array}$ \\
\hline$Z_{\mathrm{DT}}$ & $\begin{array}{l}\text { distance from the hangpoint to the } \\
\text { intercept between the monopole and the } \\
\text { line of action of drag (assumed to be the } \\
\text { centroid of area in the front view) }\end{array}$ \\
\hline$Z_{\mathrm{T}}$ & $\begin{array}{l}\text { distance from the hangpoint to the } \\
\text { intercept between the monopole and the } \\
\text { thrustline }\end{array}$ \\
\hline 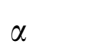 & local angle of attack \\
\hline$\theta$ & $\begin{array}{l}\text { angle between local airflow and a forward } \\
\text { perpendicular line from the hangpoint } \\
=\alpha-\phi_{\mathrm{W}}\end{array}$ \\
\hline & angle of action of thrust \\
\hline & $\begin{array}{l}\text { angle between the monopole and the earth } \\
Z \text { axes }\end{array}$ \\
\hline$\phi_{\mathrm{W}}$ & $\begin{array}{l}\text { wing control angle ( } 0 \text { places the wing } \\
\text { perpendicular to the monopole) }\end{array}$ \\
\hline$\psi$ & azimuth angle during sustained tumble \\
\hline & rotational velocity during tumble \\
\hline & rotational velocity \\
\hline
\end{tabular}

\section{INTRODUCTION}

Since their appearance in the late 1970s, weightshiftcontrolled microlight aircraft [1] have enjoyed a remarkable growth to become a large part of recreational aviation [2]. This has in part been due to their low cost and in part due to an excellent safety record [3], consistently below 30 fatal accidents per million flying hours.

References [4] to [12] report a number of accidents to weightshift-controlled microlight aircraft. With a few exceptions on specific points, these reports show a number of common factors:
1. A departure from controlled flight either following gross mishandling, flight to the stall or during flight in potentially highly turbulent conditions.

2. In most cases, the aircraft was being flown at a comparatively low weight.

3. Damage to the aircraft is consistent with very large negative $g$ overload of the wing (usually failure of the top wires and also failure downwards of the wingtips).

Evidence suggests that the wing is being forced by the total pitching moment to a very high nose-up attitude relative to the trike (impact of the basebar with the front strut will then follow, usually resulting in a failure of one of these two components, causing the propeller subsequently to impact the keel tube). Where pilots have survived the departure it is normal for them to have reported that the basebar was 'snatched from their hands' with a rate and force beyond their ability to hold it. (Note that the term 'trike' describes all of the aircraft that is not the wing or the hangbolt. The wing and trike are hinged in pitch and roll at the hangpoint, of which the hangbolt is the central component, whose removal allows the two to be separated for derigging.)

4. Autorotation of the aircraft in nose-down pitch, at a rapid rate (in excess of $360^{\circ} / \mathrm{s}$ ), followed by

5. Break-up of the aircraft in flight, preventing it from sustaining flight and usually resulting in a fatality.

There has been one previous attempt to analyse the tumble, in that case for hang-gliders, described in reference [13]. This paper does not contradict those findings, but does progress the analysis further than the previous work, briefly introducing aeroelastic and transient aerodynamic effects and analysing induced camber effects [14].

The British Microlight Aircraft Association (BMAA), in cooperation with the UK Air Accidents Investigation Branch, the UK Microlight manufacturing industry and Aerospace Engineering at the University of Southampton, has been following a programme of investigation into these similar accidents since 1997. Conclusions have been drawn concerning, firstly, the tumble mechanism and, secondly, several mechanisms by which an aircraft can enter the tumble.

\section{THE MECHANISM OF THE ESTABLISHED TUMBLE}

The tumble behaviour of a two-piece airframe like a weightshift microlight contains what initially appears to be a paradox. The tumble rotation is known to be nose down while the basebar is known to be on the front strut, a control position associated with a nose-up pitching motion in normal flight. There must therefore 
- Z axis along monopole, positive downwards.

- Origin at centre of hangbolt (centre of circle shown on diagram)

- $X$ axis perpendicular to $Z$ axis, positive forwards.

- Pitching moments and angles are positive nose-up.

- Aircraft pitching moments are calculated about the hangpoint.

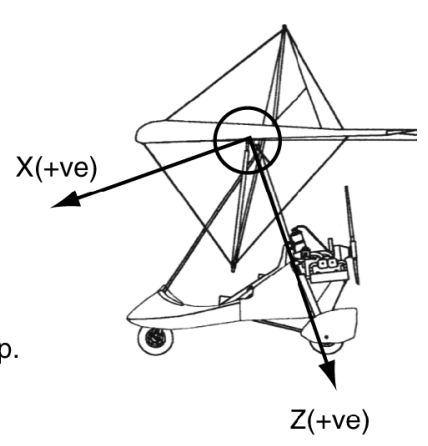

Fig. 1 Sign conventions (two-axis system, lateral axis is not used in this paper)

be some mechanism that sustains these apparently contradictory conditions.

Figure 2 shows the situation with the microlight in normal attitude and when the wing is positioned fully nose-up relative to the pilot. The centre of gravity $(\mathrm{CG})$ of the complete aircraft is also shown.

The tumble will therefore be a rotation about a lateral axis acting through a point on the aircraft close to the $\mathrm{CG}$, and the incident airflow over the wing will be as shown in Fig. 3a (A). This type of airflow is experienced with a pitching wing in normal flight, which gives rise to unsteady aerodynamic phenomena. In particular, the airflow over the leading and trailing edges of the wing are appropriate to the incident flow over a sharply cambered wing, as shown in Fig. 3a (B). This effect is known as induced camber.

Figure $3 \mathrm{~b}$ shows a photograph of an actual wingtip. As the aircraft tumbles nose-down, the inertial effects upon the wingtip trailing edge components will tend to force them in a direction from the upper to the lower surface. These trailing edge components are unlike those at the leading edge as they are not constrained by a spar

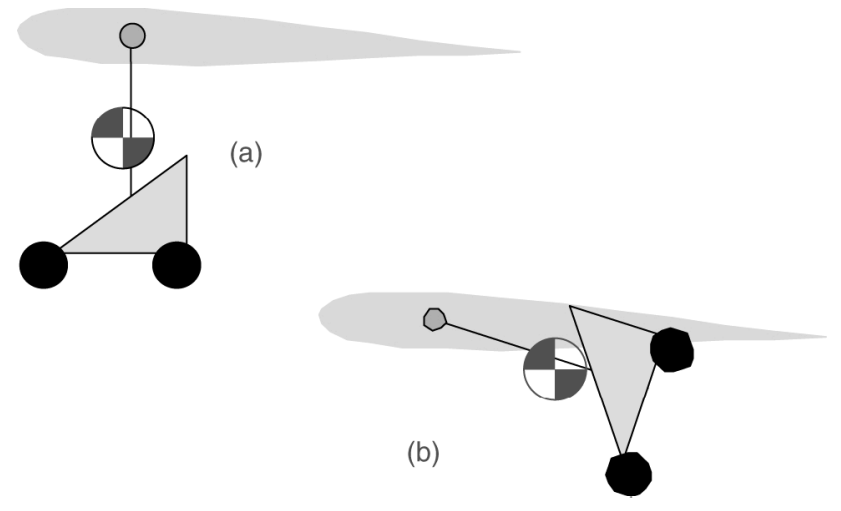

Fig. 2 Exaggerated illustration of the mass distribution of the aircraft (a) in normal flight and (b) with the basebar furthest forward, showing the approximate position of the whole aircraft $\mathrm{CG}$ (although Fig. 3b shows the 'tipstick' extending from the leading edge, which is intended to limit this movement). Tipsticks, also known as minimum washout rods, are cantilever rods protruding perpendicularly to the leading edge of the wing beneath (or occasionally within) the sail. These prevent the washout at the tips reducing below a preset value (usually about $3^{\circ}$ ) at low or negative angles of attack.

As a consequence of the nose-down rotation of the aircraft, the inertial loading will tend to deform the trailing edge structure towards the lower surface and therefore produce a localized positive camber. This will generate an additional lift in the trailing edge region which will, in turn, increase the nose-down pitching moment. This is illustrated in Fig. 4; it is also worthy of note that the wreckage of most aircraft that have suffered a tumble-related structural failure have shown failure of the wingtips, in the sense of the tip bending towards its lower surface.

There is therefore a situation where a wing-trike combination is locked into a configuration with the wing fully nose-up. Commencement of the tumble rotation causes the trailing edge panels to deflect downwards, forming some additional localized trailing edge camber through aeroelastic effects. This camber will generate aerodynamic forces, which, in turn, increase the nosedown moment. This moment, when considered with the microlight's CG location, causes the wing to rotate while translating. The wing sees the airflow as an effective camber, which therefore generate a downward lift force. Figure 4 shows the combination of these aerodynamic effects, which explains the phenomenon and the apparent paradox.

The comments about unsteady aerodynamic effects are based on existing knowledge [15] of such phenomena. However, under normal circumstances (conventional level forward flight) a wing will sense these effects as a small vertical wind perturbation superimposed on essentially forward incident airflow. With the microlight wing in a tumble a situation exists where the wing will translate and rotate but with both motions of equivalent magnitude. The aerodynamics of such a wing motion is most unusual and will require detailed tests to be undertaken to establish the flow patterns and hence an accurate prediction of the aerodynamic forces. With these data a scientific analysis of the tumble instability can be achieved. It is intended to build a wind tunnel test model of a microlight wing and to use state-of-the-art laser-based flow-measuring techniques in order to determine the wing flow in a tumble.

As a final comment, the equations of motion for the tumbling aircraft are not difficult to derive. The problem occurs when the motion is obtained by solving these equations. With the additional difficulty of the very unusual aerodynamic situation of the wing the solution will almost certainly require a numerical process to be used. 

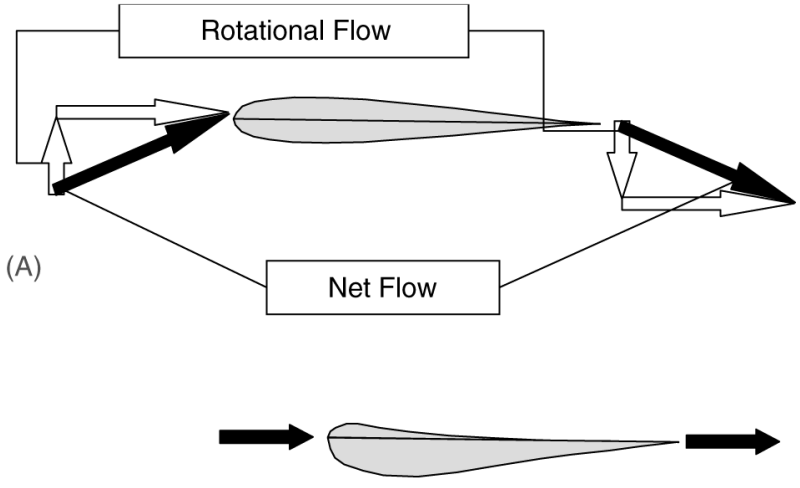

(B)

(a)

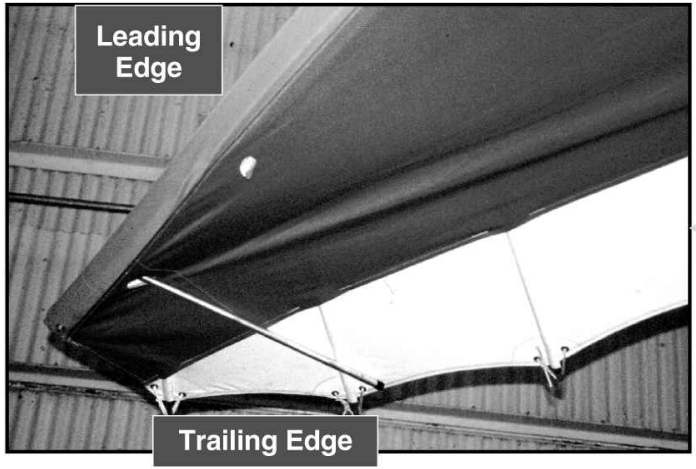

(C)

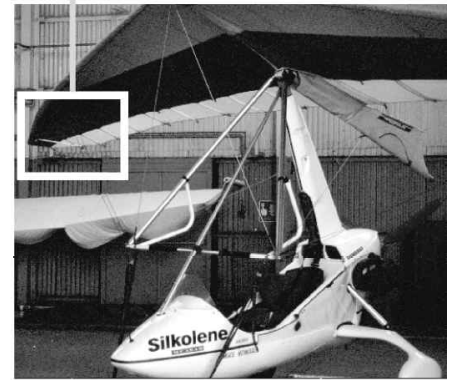

(b)

Fig. 3 (a) Illustration of induced camber during tumble. (b) Photograph of the actual wingtip from below (Pegasus Q1 wing, not under flight loads), also showing location on the aircraft

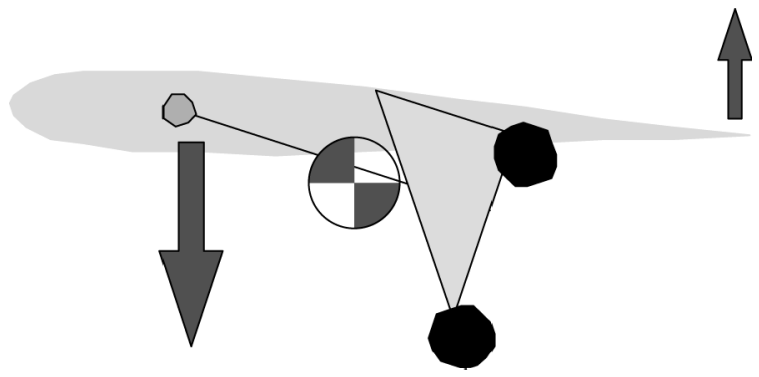

Fig. 4 Aerodynamic forces sustaining the tumble

\section{ESTIMATION OF THE MAGNITUDE OF INDUCED CAMBER DURING THE TUMBLE}

In order to assess the degree of induced camber a short analysis is presented. A wing section is fixed to an axis rotating about a point which is descending vertically. With reference to Fig. 5, an element of the wing is considered, which is distance $y$ towards the leading edge from a reference point at which a radial line (length $x$ ) from the CG of the aircraft meets the wing chord line at $90^{\circ}$. This element is rotating about the CG at a rate $\omega$ and at any given moment the line between the $\mathrm{CG}$ is perpendicular to the wing chord; the entire system direction of movement is $\psi=\omega t$. The rate of vertical, translational movement is $V$. The motion of this element may therefore be expressed by the various components of translation and rotation as indicated in Fig. 6. Using 


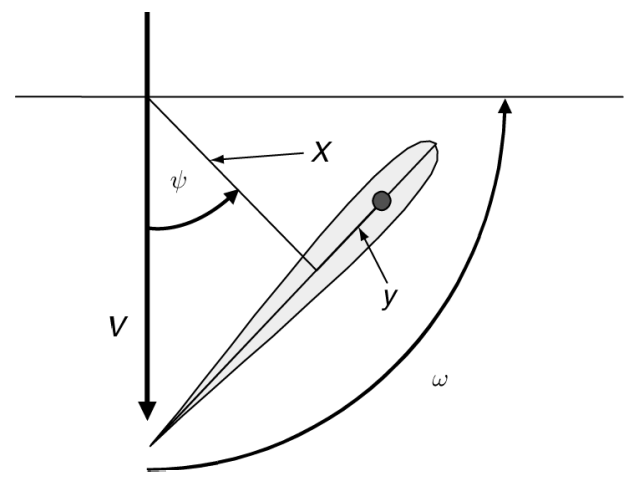

Fig. 5 Coordinates used for tumble analysis

this information, the local angle of attack (AoA) can be determined at $(x, y)$, as shown in Fig. 7.

From these results, the following expression may be written for the local AoA:

$$
\alpha=\tan ^{-1}\left(\frac{\omega y+V \cos \psi}{\omega x+V \sin \psi}\right)
$$

or

$$
\alpha=\tan ^{-1}\left(\frac{\frac{y}{x}+\frac{V}{\omega x} \cos \psi}{1+\frac{V}{\omega x} \sin \psi}\right)
$$

This can be expressed in terms of time as

$$
\alpha=\tan ^{-1}\left(\frac{\frac{y}{x}+\frac{V}{\omega x} \cos \omega t}{1+\frac{V}{\omega x} \sin \omega t}\right)
$$

From equation (2) a typical incident flow angle variation across the wing chord for the data in Table 1 is given in Table 2. This may be illustrated graphically, as shown in Fig. 8. Thus a significant induced camber effect may be seen throughout the sustained tumble.

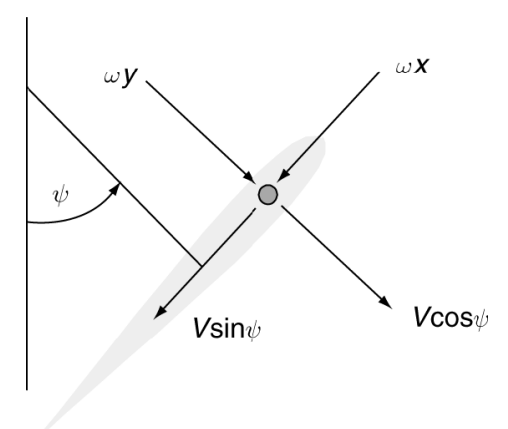

Fig. 6 Local translational and rotational velocity components of the wing element

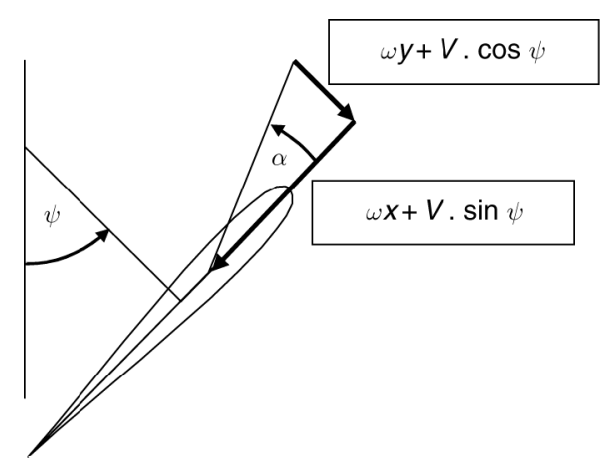

Fig. 7 Velocity components forming the local angle of attack

The 75 per cent chord result is highlighted in Fig. 8 as it is a pertinent result. Analysis of a pitching and plunging aerofoil using thin aerofoil theory [14] results in a lift force acting at the 25 per cent chord based on an incidence determined by conditions at the 75 per cent chord. In addition, a pitching moment is generated in opposition to the pitching rate and thus acts as a viscous aerodynamic damper. Figure 8 shows that for approximately three-quarters of the rotation cycle the rotation imparts a negative incidence, giving a negative lift. This negative lift force sustains the nose-down tumbling motion of the aircraft. Variation of the 75 per cent incidence shows that the pitching moment is not constant and a 'pulsing type' of rotation rate would be appropriate. (Observation of the tumbling incident described in the Appendix confirms this behaviour.) The aerodynamic damping will tend to limit the tumbling rate of the aircraft.

\section{LONGITUDINAL STATIC STABILITY OF A WEIGHTSHIFT MICROLIGHT, A MODEL DEVELOPED WITH THE INTENTION OF UNDERSTANDING THE MECHANISM OF TUMBLE INITIATION}

A weightshift-controlled microlight aeroplane is believed to have two separate and distinct longitudinal stability modes: that of the wing and that of the trike. In normal flight, when the basebar is positioned between the pilot and front strut, but touching neither, these are separate. When pitching moments are taken about the hangpoint, the aerodynamic pitching moment of the wing is balanced by the wing's own weight. Similarly, the pitching moments of the trike about the hangpoint (due to weight, drag and thrust) must sum to zero. Note that this model, which is only established for the purpose of analysing the tumble, disregards forces applied by the pilot to the basebar. This is justified because in normal (including climbing or descending) 
Table 1 Data for a typical flow angle variation

\begin{tabular}{ll}
\hline Tumble rotation rate & $400^{\circ} / \mathrm{s}$ \\
Tumble translation speed & $5 \mathrm{~m} / \mathrm{s}$ \\
Wing chord & $3 \mathrm{~m}$ \\
Perpendicular distance from the CG to the wing chord & $2.0 \mathrm{~m}$, intercepting at $0.6 \mathrm{smc}$ \\
\hline
\end{tabular}

flight pilot input to the bar will be negligible and whatever air speed the aircraft 'wishes' to adopt will normally be accepted by the pilot. Hence in most modes of flight, although the pilot will be holding the bar, little or no longitudinal force will be applied.

It is believed, mostly from the evidence of accident investigations, that the tumble occurs when some combination of conditions causes the basebar to be pushed against the front strut (this would cause the control input used by a pilot to apply the maximum nose-up pitching moment) while the sum of pitching moments upon the aircraft are strongly nose-down. Wing aerodynamic data are available from tests using the British Hang-Gliding and Paragliding Association (BHPA) test facility at Rufforth, Yorkshire (see Fig. 9). However, a theoretical model is required for the whole aircraft, which predicts the pitching moment $C_{\mathrm{M}}$ as a function of aircraft attitude. It should then be possible to combine the data for both the wing and trike in order to indicate at what combination of conditions the aircraft may continue to rotate nose-down, initiating the tumble. This may then be used to determine whether an aircraft design offers any significant risk of tumble entry, given knowledge of the wing's aerodynamic characteristics, and the desired or existing flight and manoeuvre envelope.

Consider the following model of a weightshift microlight in side view (see Fig. 10), disregarding for the time being the aerodynamic pitching moment of the wing. All pitching moments will be taken about the hangpoint. This model is shown in Fig. 11 and makes use of the following definitions and assumptions.

\subsection{Definitions}

1. $W_{\mathrm{T}}$ is the weight of the trike. As shown in Fig. 12, it acts through the trike $\mathrm{CG}$, which is located $Z_{\mathrm{CGT}}$ below the hangpoint (in a direction parallel to the monopole) and $X_{\mathrm{CGT}}$ forward of the monopole axis (in a direction perpendicular to the monopole). The weight acts at an angle $\phi_{\mathrm{g}}$ relative to the monopole axis; $\phi_{\mathrm{g}}=0$ when the monopole is perpendicular to the surface of the earth, increasing with the aircraft's nose-up attitude.

2. $D_{\mathrm{T}}$ is the drag due to the trike. It acts through the monopole at a point $Z_{\mathrm{DT}}$ below the hangpoint and at an angle $\theta$ relative to a perpendicular to the monopole such that if $\theta=0$ the monopole is perpendicular to the relative airflow and if $\theta$ is positive the monopole is more nose-up.

3. $T$ is the thrust due to the engine. It acts through the monopole at a point $Z_{\mathrm{T}}$ below the hangpoint and at an angle $\theta_{\mathrm{T}}$ relative to a perpendicular to the monopole such that if $\theta_{\mathrm{T}}=0$ the thrustline is perpendicular to the monopole and if $\theta_{\mathrm{T}}$ is positive the thrustline becomes more nose-up.

4. $W_{\mathrm{W}}$ is the weight of the wing. It acts through the wing $C G$ which is on the wing keel a distance $L_{\mathrm{CGW}}$ behind the hangpoint. The wing itself is at an angle $\phi_{\mathrm{W}}$ nose-up compared to a perpendicular to the monopole. The weight acts at an angle $\phi_{\mathrm{g}}$ relative to the monopole axis; $\phi_{\mathrm{g}}=0$ when the monopole is perpendicular to the surface of the earth, becoming more positive with the aircraft's attitude increasing nose-up.

Table 2 Local angle of attack (deg)

\begin{tabular}{lllllll}
\hline & \multicolumn{6}{c}{ Chordwise station (\%) } \\
\cline { 2 - 7 } Azimuth angle (deg) & 0 & 20 & \multicolumn{1}{c}{40} & \multicolumn{1}{c}{60} & \multicolumn{1}{c}{80} & 100 \\
\hline 0 & 45.82 & 36.09 & 23.22 & 7.35 & -9.71 & -25.22 \\
30 & 36.68 & 27.65 & 16.86 & 4.70 & -7.90 & -19.78 \\
60 & 30.76 & 22.30 & 12.68 & 2.28 & -8.27 & -18.29 \\
90 & 27.67 & 19.27 & 9.92 & 0.00 & -9.92 & -19.27 \\
120 & 27.28 & 18.29 & 8.27 & -2.28 & -12.68 & -22.30 \\
150 & 30.13 & 19.78 & 7.90 & -4.70 & -16.86 & -27.65 \\
180 & 37.64 & 25.22 & 9.71 & -7.35 & -23.22 & -36.09 \\
210 & 50.85 & 37.26 & 16.35 & -9.87 & -32.67 & -47.95 \\
240 & 65.56 & 54.66 & 31.81 & -9.63 & -43.82 & -60.25 \\
270 & 72.50 & 64.69 & 46.59 & 0.00 & -46.59 & -64.69 \\
300 & 68.51 & 60.25 & 43.82 & 9.63 & -31.81 & -54.66 \\
330 & 57.60 & 47.95 & 32.67 & 9.87 & -16.35 & -37.26 \\
360 & 45.82 & 36.09 & 23.22 & 7.35 & -9.71 & -25.22 \\
\hline
\end{tabular}


Inflow Angles during Tumble Rotation

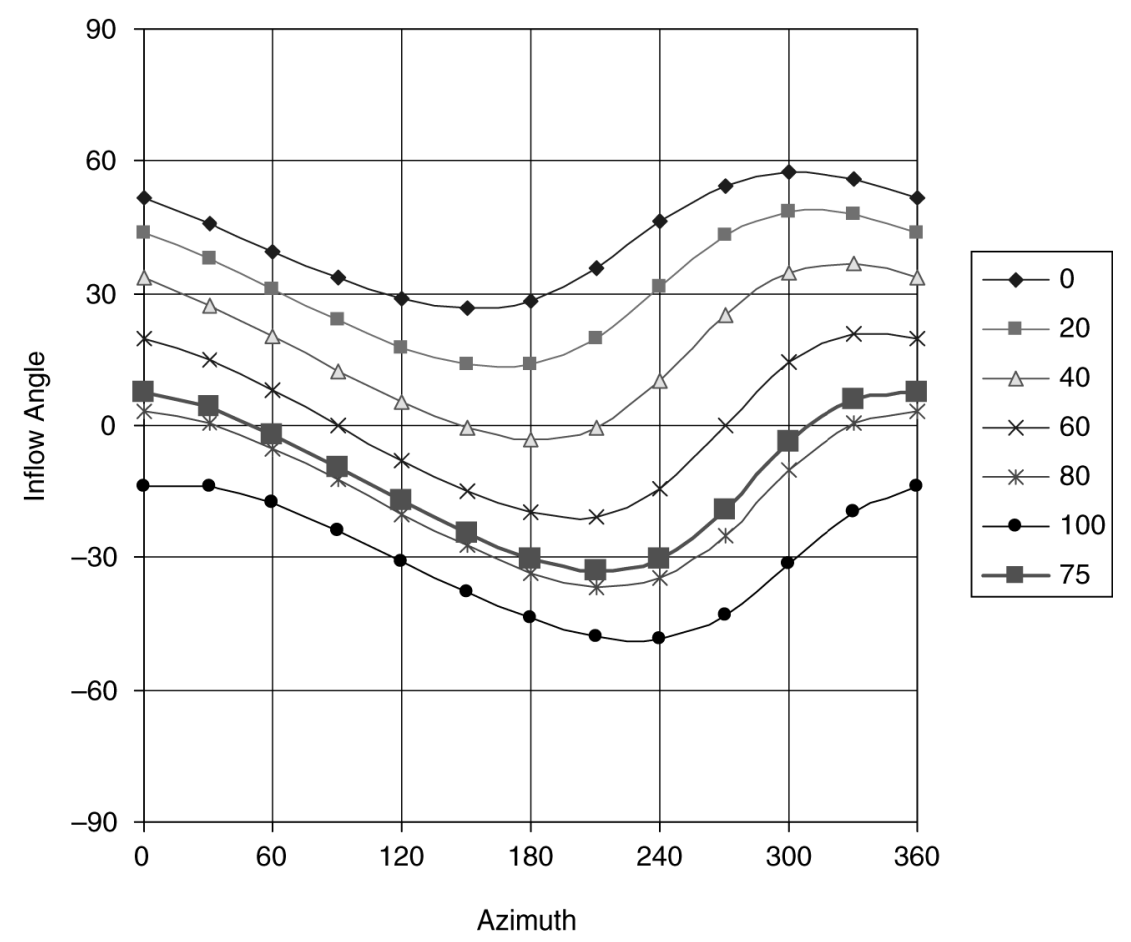

Fig. 8 Local angle of attack variation

5. $L$ is the lift due to the wing. It acts through the hangpoint at an angle perpendicular to the wing and is positive when acting towards the upper surface of the wing.

6. $D_{\mathrm{W}}$ is the drag due to the wing. It acts through the hangpoint in a direction parallel to the wing keel and is positive when acting towards the trailing edge.

\subsection{Assumptions}

1. The aircraft is in an unaccelerated state.

2. Trike drag acts in the wind axis.

3. Wing lift and drag act at the hangpoint.

4. The pilot has lost control of the wing and therefore provides no input.

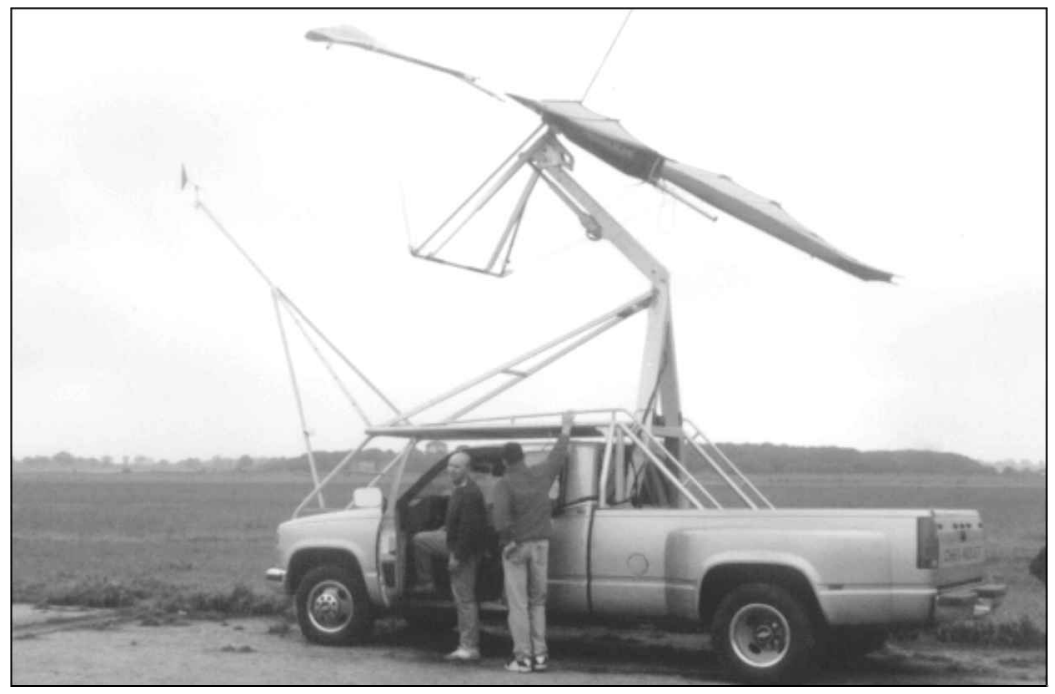

Fig. 9 BHPA hang-glider test facility at Rufforth, Yorkshire 


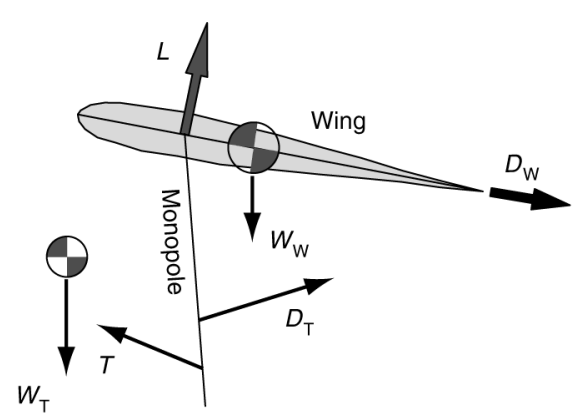

(a)

Fig. 10 (a) Forces, distances and angles relevant to the longitudinal stability model. (b) Dimensions and angles of the theoretical model
The moments in pitch about the hangbolt acting on the whole aircraft are as follows (except for the wing aerodynamic pitching moments):

1. The moment due to the wing aerodynamic pitching moment is disregarded in this analysis, for reasons explained above.

2. The moment due to the effect of wing lift is taken to be zero, since lift is considered to act through the hangpoint.

3. The moment due to the effect of wing drag is taken to be zero, since wing drag is considered to act through the hangpoint.

4. The moment due to the effect of wing weight is

$$
W_{\mathrm{W}} L_{\mathrm{CGW}} \cos \left(\phi_{\mathrm{W}}-\phi_{\mathrm{g}}\right)
$$

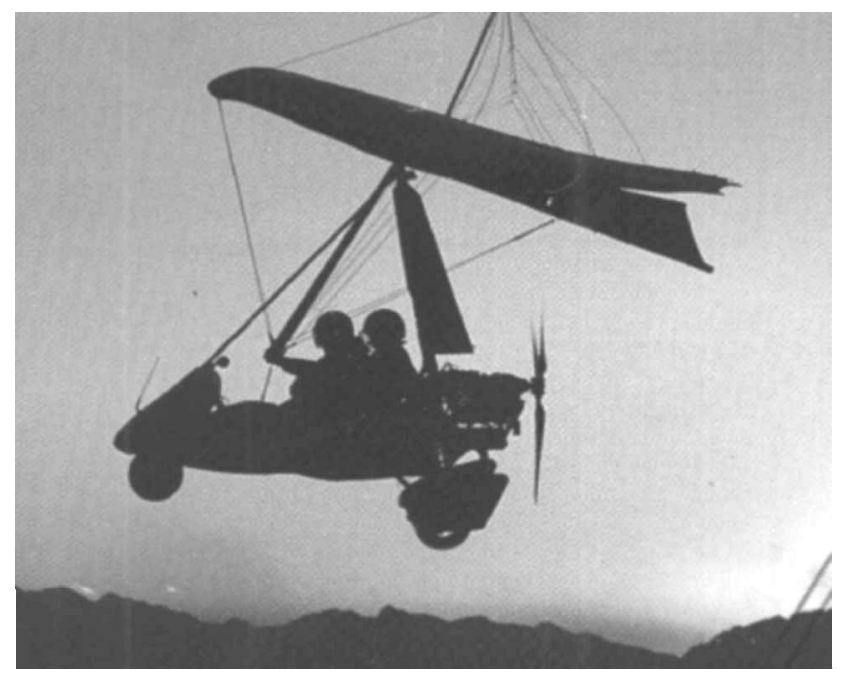

Fig. 11 Illustration of Pegasus Quantum 15-912 in a typical orientation (illustrated in Fig. 10)
5. The moment due to the drag of the trike is

$$
-D_{\mathrm{T}} Z_{\mathrm{DT}} \cos \theta=-K_{\mathrm{DT}} V^{2} Z_{\mathrm{DT}} \cos \theta
$$

6. The moment due to thrust is

$$
T Z_{\mathrm{T}} \cos \phi_{\mathrm{T}}
$$

7. The moment due to the weight of the trike (see Fig. 12) is

$$
W_{\mathrm{T}}\left(-X_{\mathrm{CGT}} \cos \phi_{\mathrm{g}}+Z_{\mathrm{CGT}} \sin \phi_{\mathrm{g}}\right)
$$

Summing these components, the total pitching moment acting upon the aircraft is given by

$$
\begin{aligned}
M_{\mathrm{TOTAL}}= & W_{\mathrm{W}} L_{\mathrm{CGW}} \cos \left(\phi_{\mathrm{W}}-\phi_{\mathrm{g}}\right) \\
& -K_{\mathrm{DT}} V^{2} Z_{\mathrm{DT}} \cos \theta \\
& +T Z_{\mathrm{T}} \cos \phi_{\mathrm{T}} \\
& +W_{\mathrm{T}}\left(-X_{\mathrm{CGT}} \cos \phi_{\mathrm{g}}+Z_{\mathrm{CGT}} \sin \phi_{\mathrm{g}}\right)
\end{aligned}
$$

However, since it is difficult to predict the value of

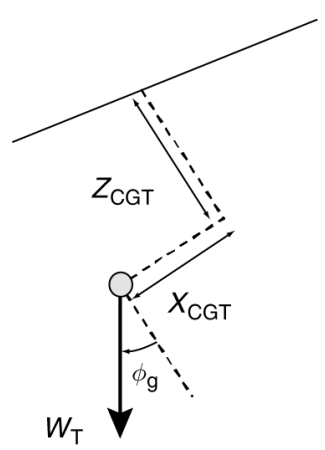

Fig. 12 Coordinates of the trike CG relative to the hangpoint 
thrust during a departure from controlled flight, and also the effect of thrust is to pitch the aircraft nose-up, when considering the risk of a nose-down departure, zero thrust will be regarded as the worst case. Therefore, it is conservative and appropriate to disregard it from the above formula, which is simplified further to

$$
\begin{aligned}
M_{\mathrm{TOTAL}}= & W_{\mathrm{W}} L_{\mathrm{CGW}} \cos \left(\phi_{\mathrm{W}}-\phi_{\mathrm{g}}\right) \\
& -K_{\mathrm{DT}} V^{2} Z_{\mathrm{DT}} \cos \theta \\
& +W_{\mathrm{T}}\left(-X_{\mathrm{CGT}} \cos \phi_{\mathrm{g}}+Z_{\mathrm{CGT}} \sin \phi_{\mathrm{g}}\right)
\end{aligned}
$$

This formula may then be used to estimate the total pitching moment on the trike. The input variables required to determine $M_{\mathrm{TOTAL}}$ are as follows:

1. $W_{\mathrm{W}}$ and $W_{\mathrm{T}}$ are basic design values of the aircraft. However, it should be borne in mind that while for a specific type $W_{\mathrm{W}}$ is fixed, $W_{\mathrm{T}}$ will vary according to occupancy and fuel state. However, for any given type, the minimum and maximum permitted loadings are published.

2. $Z_{\mathrm{DT}}, \theta_{\mathrm{T}}, X_{\mathrm{CGT}}, Z_{\mathrm{CGT}}$ and $K_{\mathrm{DT}}$ are functions of aircraft geometry and may be obtained from design data.

3. $\phi_{\mathrm{W}}, \phi_{\mathrm{g}}$ and $\theta$ are flight variables. It is known that typically in the cruise $\phi_{\mathrm{g}} \approx-15^{\circ}$ and $\phi_{W} \approx 30^{\circ}$. The range of values of $\phi_{\mathrm{W}}$ will be approximately $\pm 10^{\circ}$ compared with this value. Also, normal (and usually placarded) operating limitations for an aircraft in this class are $\pm 30^{\circ}$ pitch attitude, compared to the normal level flight attitude. Therefore, it may be considered that during flight within the normal envelope, $-45^{\circ}<\phi_{g}<15^{\circ}$ and $20^{\circ}<\phi_{\mathrm{W}}<40^{\circ}$.

For the purposes of modelling, it is appropriate to examine a wider range of values of $\phi_{\mathrm{g}}$ than might be experienced within the normal envelope, so values of $-105^{\circ}<\phi_{\mathrm{g}}<75^{\circ}$ will be considered (equating to attitudes between vertically upwards and vertically downwards, as seen by the pilot). The significant case is the one where the pilot would not be able to prevent a nose-down departure. Assuming then a full nose-up pitch inceptor input, it can be further assumed that $\phi_{\mathrm{W}}=40^{\circ}$.

4. $\theta$, the trike angle of attack, is relevant insofar as the drag due to the trike acts in a nose-down direction. Therefore it will be considered to be $0^{\circ}$, again since this is the worst case for a nose-down pitching departure.

In such a case, a polar is required:

$$
\begin{aligned}
M_{\mathrm{TOTAL}}= & W_{\mathrm{W}} L_{\mathrm{CGW}} \cos 40^{\circ} \cos \phi_{\mathrm{g}} \\
& -K_{\mathrm{DT}} V^{2} Z_{\mathrm{DT}} \cos 0^{\circ} \\
& -W_{\mathrm{T}} \sqrt{X_{\mathrm{CGT}}^{2}+Z_{\mathrm{CGT}}^{2}} \\
& \times \sin \left[\phi_{\mathrm{g}}-\sin ^{-1}\left(\frac{X_{\mathrm{CGT}}}{Z_{\mathrm{CGT}}}\right)\right]
\end{aligned}
$$

However, for the purposes of considering the trike alone, the first term of this equation is omitted.

Figure 13 shows the value of $M_{\text {TOTAL }}$ as a function of $\phi_{\mathrm{g}}$ for a Mainair Gemini trike (omitting the $W_{\mathrm{W}}$ term) for both its maximum and minimum permitted loadings. $V$ is assumed to be 43 knots, since this is a typical cruising air speed, and also the speed around which the best quality wing aerodynamic test data are available. This may be compared to the graph in Fig. 14 for a correctly adjusted Mainair Flash 2 alpha wing, which might typically be fitted to this trike.

If the wing is pushed through the stalling angle of attack to about $25^{\circ} \mathrm{AoA}$, then the aerodynamic pitching moment will be about $600 \mathrm{Nm}$ nose-up, as seen in Fig. 14. However, if the wing is considered, this pitching moment is reached at about $20^{\circ}$ nose-up, regardless of weight (which equates approximately to $35^{\circ}$ nose-up as seen by the pilot). If the aircraft was stalled at a greater nose-up pitch attitude of, for example, $30^{\circ}$ nose-up $\left(45^{\circ}\right.$ as seen by the pilot) then while the wing pitching moment will remain at about $600 \mathrm{Nm}$ the trike, depending upon weight, will have a pitching moment of $1000-1500 \mathrm{Nm}$ nose-down. This will, once the basebar has been touched by the front strut (creating a rigid system), force the whole aircraft, in a rigid state, nose-down, rotating about the whole aircraft CG, which will, due to the relative masses, be close to the trike CG. The effect of this, as previously discussed, is to induce an apparent reverse camber at the wing (see Fig. 15). This induced reverse camber is likely to cause a reversal in pitch stability and thus a tendency to further pitch down. A negative lift force will also 'lock' the trike to the aircraft, maintaining the rigid system.

The BMAA, in association with the UK Civil Aviation Authority and the UK microlight manufacturing industry, is currently studying the approach detailed above with the intent of developing pass/fail criteria for new or suspect aircraft designs. Once tested in sample certification programmes, it is intended that this will ultimately be incorporated into the safety requirements for this class of aircraft [16].

\section{AVOIDING THE TUMBLE}

The analysis above indicates that a tumble can potentially occur if the aircraft enters a flight condition where the nose-down pitching moment due to the weight of the trike is greater than that of the pitching moment of the wing, locking the trike to the wing and thereby forcing the entire aircraft to pitch nose-down as a rigid body. This may be entered with the aid of engine thrust, creating this situation when thrust is lost either deliberately (through throttle closure) or inadvertently (through engine failure). 


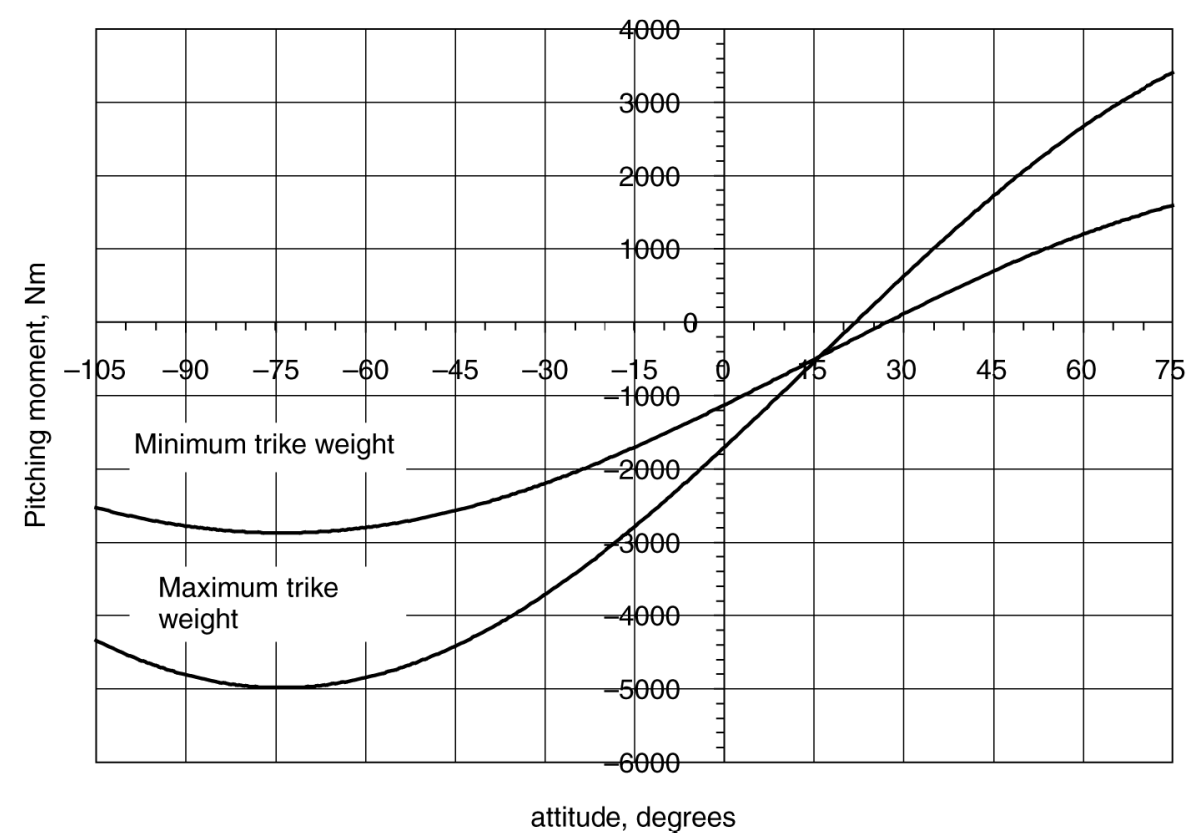

Fig. 13 Pitching moment of the trike about the hangpoint, zero thrust

Using the simple model of the trike longitudinal stability given above, added to the aerodynamic characteristics of the trike, it becomes possible to predict the conditions at which the tumble might occur. It should be borne in mind that the tumble might not necessarily occur, since the rate of pitching must be sufficient to cause the inverse camber on the wing that is associated with the sustained tumble.

From this analysis, the tumble appears to be a function of both the wing and trike characteristics. A trike with high weight or a long monopole will have a greater pitching moment at a steep nose-up attitude, and therefore a greater tendency to tumble.

\section{THE EFFECT OF WEIGHT ON THE TUMBLE}

It is known from the history of tumble accidents that the more highly loaded the trike is the less the aircraft will tend to tumble. At first sight of the graph in Fig. 13, this does not make sense. However, once the nose-down

F2a, G-MVEP, Tipsticks fitted, Correct Lufflines, 44 +/-2.7 kn

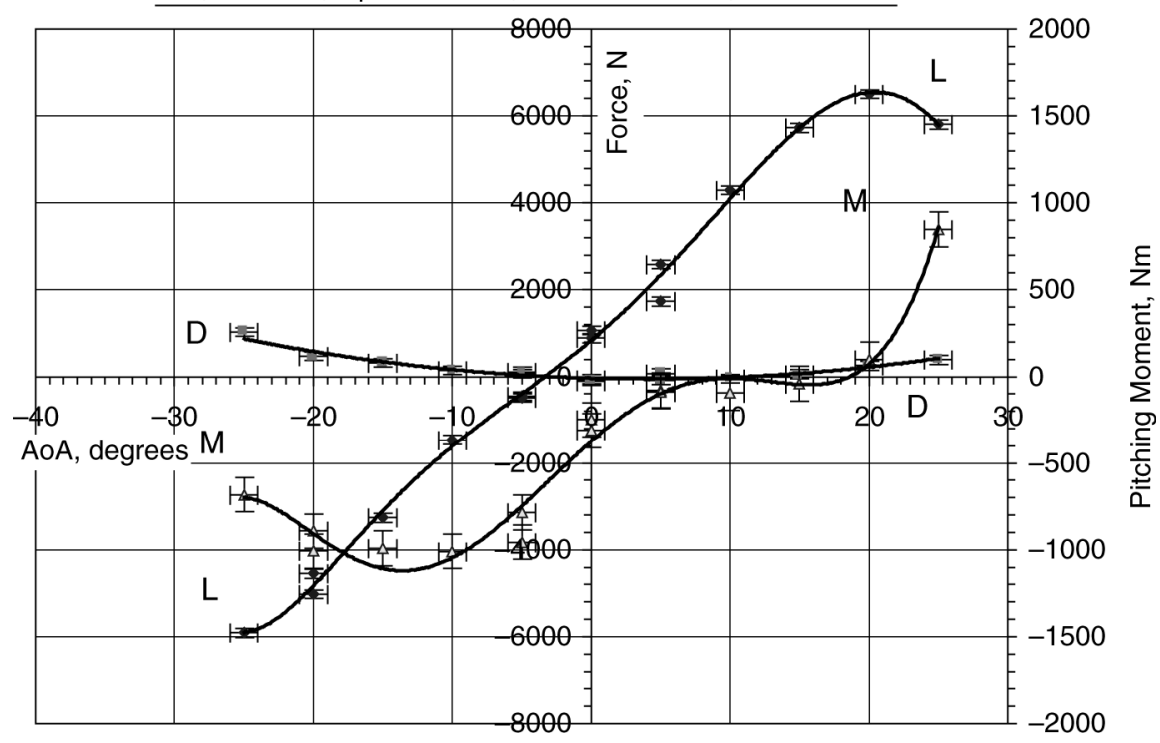

Fig. 14 Characteristics of Mainair Flash 2 alpha wing 


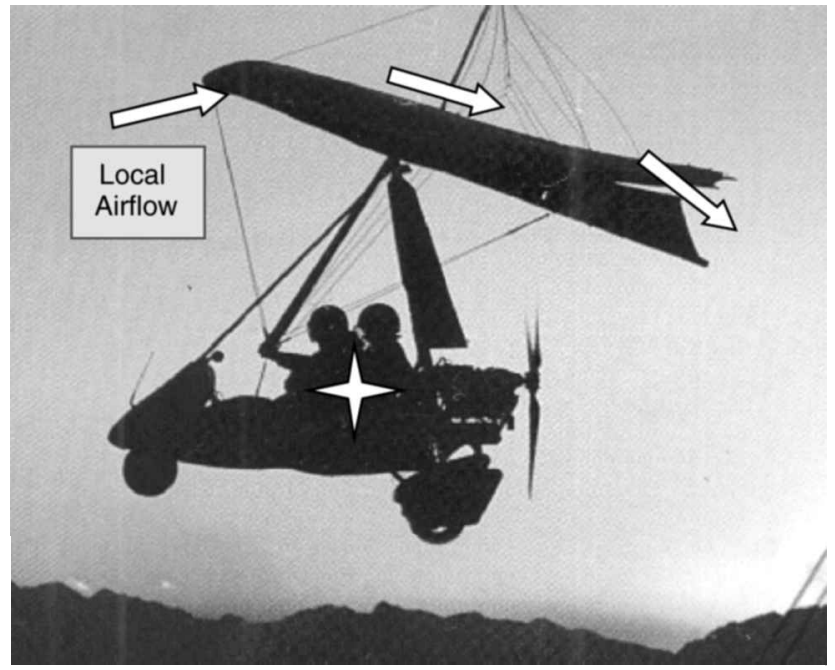

Fig. 15 Illustration of induced flow superimposed upon the aircraft image

pitch departure occurs, a more lightly loaded trike will result in a higher $\mathrm{CG}$ and thus a greater angle of inflow into the wing. Therefore, for a lower trike mass, the induced camber at the wing will be greater since the point of rotation will be closer to the wing. Also at a lower trike mass, the rotational inertia in pitch will be less.

For example, a microlight aircraft wing mass of $50 \mathrm{~kg}$, a trike mass of $150 \mathrm{~kg}$ and a distance between these CGs of $1.5 \mathrm{~m}$ gives a total rotational inertia of $87.5 \mathrm{~kg} \mathrm{~m}^{2}$. The wing is $1 \mathrm{~m}$ from the aircraft CG. With a trike mass of $300 \mathrm{~kg}$, the rotational inertia is $96.5 \mathrm{~kg} \mathrm{~m}^{2}$ and the wing is $1.3 \mathrm{~m}$ from the aircraft CG. Thus, with the same pitching moment applied, and disregarding aerodynamic damping (at present, data is not of sufficient quality), the effect of a net nose-down pitching moment of $500 \mathrm{Nm}$ can be ascertained by the simple analysis given in Table 3.

It is thus demonstrated that a reduced trike weight will result in a significantly greater induced camber following an aircraft stall at a high nose-up attitude. Therefore, the risk of the sustained tumble occurring following a nose-up stall is considerably greater.

\section{EFFECT OF WING SETTINGS}

Experience has also shown that a mis-rigged wing, particularly one in which the luffline tension is insufficient, will display a greater tendency to tumble. Figure 16 shows four curves for the Mainair Flash 2 alpha wing (see Fig. 14) already discussed, for a variety of conditions. These plots vary in three ways, none of which impact significantly upon the discussion above.

The wings with insufficient luffline tension display a flat curve (indicating very low apparent longitudinal static stability) around the trim. Without either tipsticks or correct luffline tension, the wing displays a pitch stability minimum about zero AoA. It is believed, from previous work by Kilkenny [17] on hang-glider stability and from discussions with microlight wing designers, that this is related to the luffing dive (a mode of flight where unsatisfactory longitudinal stability characteristics cause a constant speed or accelerating descent which is usually unrecoverable) and not to the tumble.

While all wings display an apparent tendency towards a nose-down pitching moment at very low angles of attack (well below anything likely to be experienced within the permitted manoeuvre envelope), this occurs at a higher angle of attack for a wing without tipsticks and with incorrect luffline tension. (Note that the lack of data at lower angles of attack than those shown in Fig. 16 is due to a physical limitation of the BHPA test rig. The only other known facility in the world (located in Germany) is of similar design and thus at present there is no means of finding out exactly what happens at these angles of attack.)

It is proposed that this last characteristic is significant to tumble initiation. While it is, with the current state of knowledge, only possible to conjecture as to exactly what happens to the forces and moments acting upon the wing during the initial pitch-down of tumble initiation, it is a reasonable assumption that the misrigged wing shown by curve (4) in Fig. 16 will show a greater tendency to pitch-down as the reduction in AoA occurs than the correctly rigged wing (irrespective of any induced reverse camber). In surveying these plots, it appears that the correct luffline tension is important in preventing the tumble, but the presence of tipsticks

Table 3 Effect of trike mass upon inflow angle for a constant pitching moment

\begin{tabular}{lcc}
\hline & Lighter aircraft & Heavier aircraft \\
\hline Total rotational inertia $\left(\mathrm{kg} \mathrm{m}^{2}\right)$ & 87.5 & 96.5 \\
Rotational acceleration, assuming a $500 \mathrm{~N} \mathrm{~m}$ nose-down pitching moment and no aerodynamic & 5.73 & 5.18 \\
$\quad$ damping $(\mathrm{rad} / \mathrm{s})$ & 328 & 296 \\
Resultant rotational velocity after $1 \mathrm{~s}, \omega(\mathrm{deg} / \mathrm{s})$ & 9.38 & 7.23 \\
Downward velocity of the nose, assuming it is a nominal $1 \mathrm{~m}$ in front of the hangpoint $(\mathrm{m} / \mathrm{s})$ & 15 & 18 \\
Nominal aircraft stalling speed at this weight $\approx V_{\text {So }} \sqrt{\mathrm{MTOW} / W}(\mathrm{~m} / \mathrm{s})$ & 32 & 21 \\
Approximate angle of resultant flow, at the wing leading edge, at stalling speed $(\mathrm{deg})$ & \\
\hline
\end{tabular}




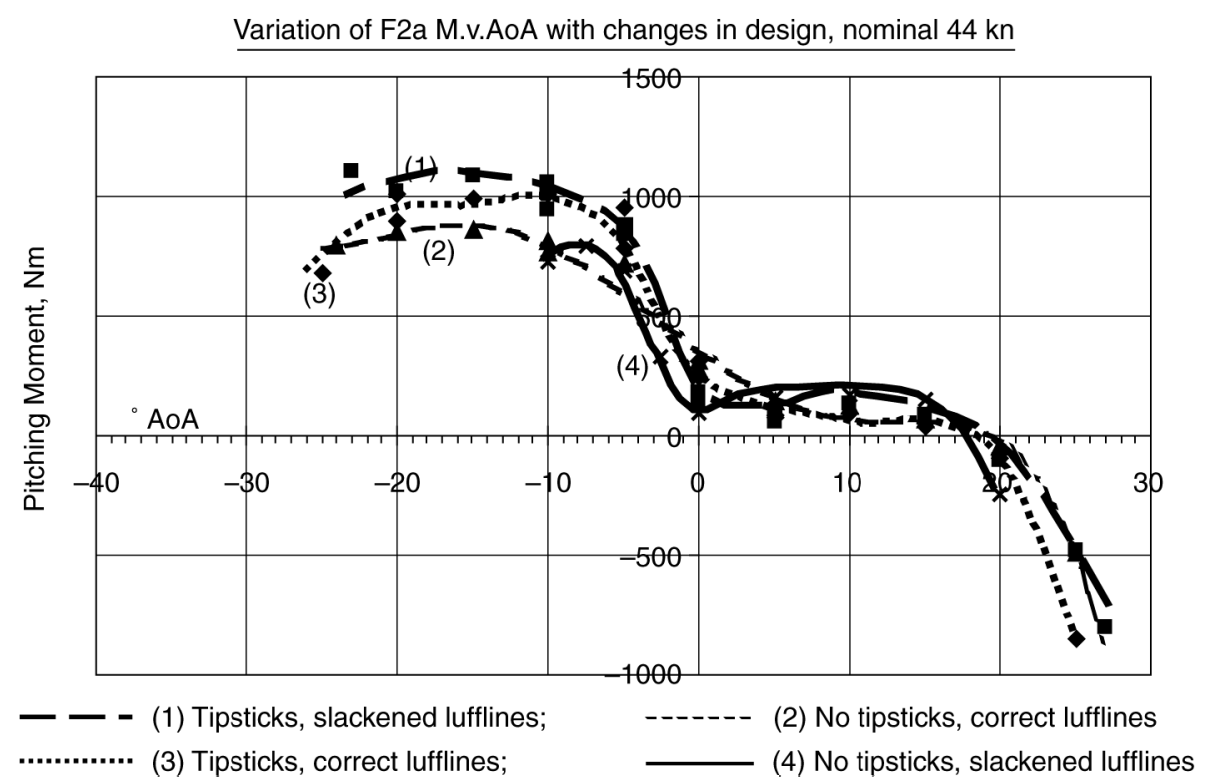

Fig. 16 Effect of different rigging conditions upon Mainair Flash 2 alpha wing

provides a valuable backup - if luffline tension ceases to be correct. In this situation, the tipsticks appear likely to maintain a large margin between the normal cruise condition and the normal flying range of positive angles of attack. Figure 16 gives reasonable grounds to believe that pitch stability reversal will occur at angles of attack above $-20^{\circ}$, which the previous analysis indicates might potentially occur with sufficient mishandling in a lightweight aircraft.

\section{INITIATION OF THE TUMBLE}

The description of the tumble initiation above shows that for the tumble to occur, the aircraft must be steeply nose-up with a low throttle setting or failed engine. There are several ways in which this might occur, which are discussed below:-

\subsection{Cause 1: the 'whip-stall'}

The whip-stall is caused by an aggressive entry (at a high deceleration rate, well in excess of the $1 \mathrm{kn} / \mathrm{s}$ normally recommended) to the aerodynamic stall, followed by an equally aggressive recovery initiation by the pilot (pulling in the control bar rapidly). This is a manoeuvre which may be used by test pilots (with great care) to allow them to demonstrate $V_{\mathrm{NE}}$ or $V_{\mathrm{DF}}$ in this class of aircraft [18], which are otherwise control limited and unable to demonstrate high-speed flight for certification purposes. However, there is absolutely no need for a private pilot to ever carry out this manoeuvre in normal flight; the whip-stall is specifically prohibited by all
Microlight manufacturers and by the UK pilot training syllabus [19]. It is considered likely (and several eyewitness reports of fatal accidents bear this outmost recently the October 2000 fatality to a Pegasus Quantum) that this mechanism can lead to the tumble.

The sequence of actions in the whip stall is detailed below:

1. The pilot places the aircraft in a climbing attitude and pushes the control bar out rapidly to achieve a high deceleration rate. At the steepest possible noseup attitude, the throttle is closed.

2. The air speed decreases rapidly, with unsteady aerodynamic characteristics delaying the onset of aerodynamic stall until a lower air speed is achieved than would normally be experienced.

3. At the point of stall, the wing's aerodynamic pitching moment becomes strongly nose-down. Due to the low air speed, this is likely to be less than if the stalling angle of attack is reached in a less dynamic manoeuvre.

4. The trike pitches down and pushes against the wing (with the front strut against the basebar), creating a rigid system upon which a net nose-down pitching moment is acting.

\subsection{Cause 2: combined spiral instability combined with a loss of horizon}

Weightshift microlight aircraft are approved in all countries of which the authors have knowledge, but only for flight in visual meteorological conditions (VMC). This implies a guaranteed visual horizon which the pilot may use as a reference when correcting small 
rolling departures (such as may be caused by temporary inattention or by turbulence). However, it is possible through ill-luck or poor judgement for an aircraft to enter instrument meteorological conditions (IMC), where a defined horizon cannot be guaranteed (such as in cloud). If this happens, any pilot will attempt to remove themselves and their aircraft from this as quickly as possible; however, if the pilot is unable to extract the aircraft from this situation it is almost inevitable that some cause (most likely the turbulence commonly found inside or near to most clouds) will initiate an undemanded rolling manoeuvre. Unlike most conventional aeroplanes, most weightshift microlight aircraft are spirally unstable; thus, an initial small bank angle is likely to increase without (unless a horizon reference is available) the pilot's knowledge or ability to control it. The aircraft would then enter a slow roll, potentially through $90^{\circ}$ of bank to a condition where the pendulum stability that keeps the trike below the wing ceases to act - inevitably causing some loss of control. It is then possible that the aircraft will find itself in an unsustainably steep nose-up attitude. It is noticeable that some tumble accident reports, particularly those relating to the G-MVEP, have occurred in conditions where the horizon was known to be poor and where the subsequent damage to the aircraft showed that the basebar had fractured (in contact with the front strut) at the end, rather than in the centre as would be implied by a symmetric manoeuvre. This implies a rolling component to the departure from controlled flight, which would be consistent with this mechanism.

Table 4 shows the results of a brief test carried out to demonstrate the spiral instability of a weightshift microlight aircraft. A Raven-X weightshift microlight, flown solo, was trimmed in moderately turbulent air conditions and the controls released. The resultant bank angle was estimated based upon a visual horizon, and the time to reach these given bank angles was measured. This demonstrates that following flight into IMC such a departure could readily happen within $60 \mathrm{~s}$ (obviously, the presence of spiral instability will vary between aircraft types and different power settings). The test aircraft is illustrated in Fig. 17.

In the case of the G-MVEP, referred to above, it would be a reasonable deduction that having lost the visual horizon the pilot (who was still under training) might have rolled beyond permissible limits in under $60 \mathrm{~s}$.

\subsection{Cause 3: failed loop manoeuvre}

While weightshift microlight aircraft are neither approved, nor should be, for aerobatics, it is occasionally known for a pilot to attempt aerobatic manoeuvres. There are several reported instances of pilots attempting to conduct a loop in such an aircraft. If positive normal acceleration is maintained throughout this manoeuvre it can be executed as safely as in any other aircraft. However, as with any other aircraft, if the aircraft runs out of energy near to the top of the loop, then the pilot finds himself inverted without sufficient air speed to complete the manoeuvre. In this case, the inevitable consequence will be a negative angle of attack, leading to a tumble. The Appendix shows a sequence of frames from a film taken of a French Cosmos aircraft. The aircraft was flying an air display sequence that included a loop, which failed. The result was a tumble resulting in the aircraft's destruction and death of the pilot on collision with the ground.

\subsection{Cause 4: flight through own wake vortex}

It is well known that a minimum safe separation should be ensured between landing aircraft, particularly larger aircraft, which tend to generate very large vortex wakes that can normally be expected to remain for up to $80 \mathrm{~s}$ $[\mathbf{2 0}, \mathbf{2 1}]$ in normal conditions, rather longer in very still air. The weightshift microlight, using as it does a delta wing, tends to generate a particularly large wake vortex for the size of the aircraft and is capable of generating a considerable upset [22]. For this reason, pilots of

Table 4 Test to demonstrate weightshift spiral instability

$\begin{array}{ll}\text { (a) aircraft and atmospheric conditions } \\ \text { Aircraft } & \text { Southdown Raven-X (Rotax } 447 \text { engine) }+60 \text { inch 3-blade Ivoprop propeller at } 9^{\circ} \text { pitch (propeller approved by } \\ & \text { MAAN 1076) } \\ \text { Registration } & \text { G-MNKZ } \\ \text { Crew } & \text { Gratton (solo) } \\ \text { Conditions } & \text { CAVOK, light turbulence, nil Wx, OAT }+5^{\circ} \mathrm{C}, \text { No. } 3 \text { from front hangpoint setting giving } 48 \text { mile } / \mathrm{h} \text { IAS trim } \\ \text { Date } & 13 \text { February } 2001 \\ \text { Test } & \text { Aircraft flown in light but perceptible turbulence over woodland, nominal } 1000 \mathrm{ft} \text { on } \mathrm{QFE} 1024 \mathrm{hPa}\end{array}$

(b) Test results

\begin{tabular}{llll}
\hline Power $(\mathrm{r} / \mathrm{min})$ & Time at $30^{\circ}$ bank $(\mathrm{s})$ & Time at $45^{\circ}$ bank $(\mathrm{s})$ & Time at $60^{\circ}$ bank $(\mathrm{s})$ \\
\hline 3000 (light idle) & 25 & 40 & Test abandoned due to ground proximity \\
5000 (PLF) & 10 & 20 & 25 \\
$6500(\mathrm{MCP})$ & 10 & 15 & 20 \\
\hline
\end{tabular}




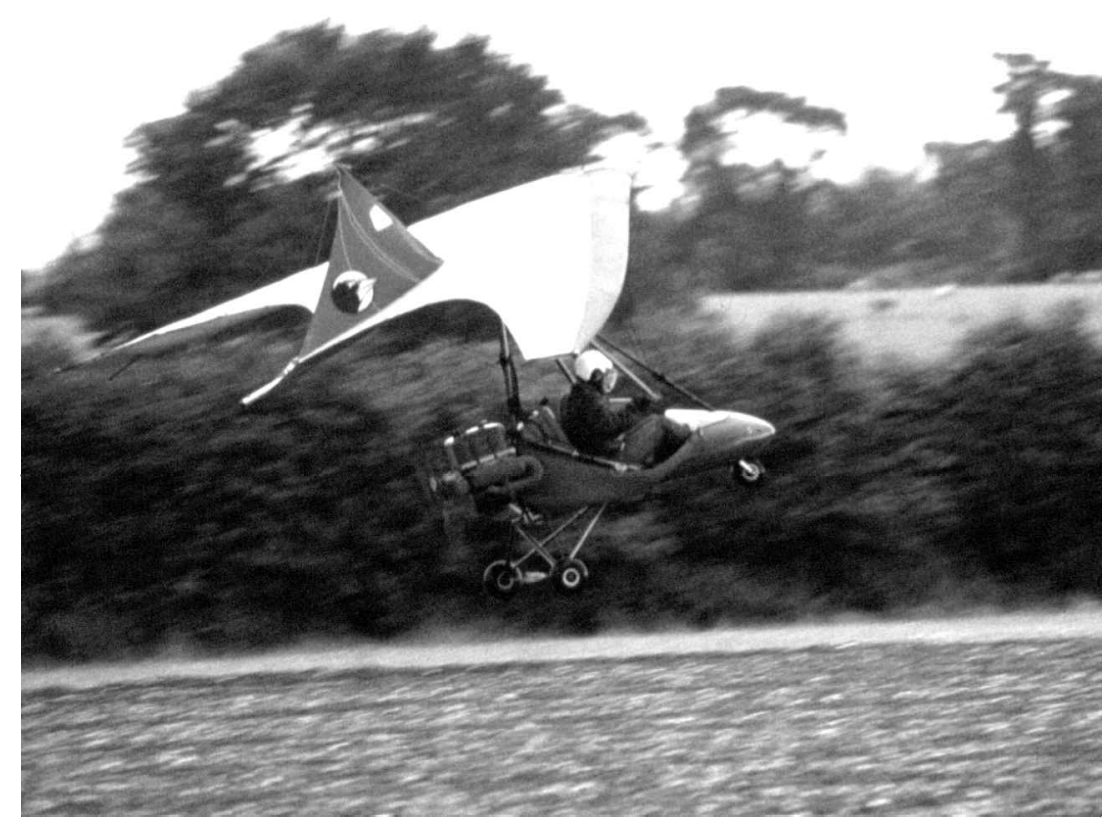

Fig. 17 Southdown Raven-X G-MNKZ

weightshift aircraft are taught that level turns should never be continued beyond $270^{\circ}$ and preferably not beyond $180^{\circ}$ without climbing or descending during the turn.

Considering a typical turning manoeuvre at $45 \mathrm{kn}$ CAS, $60^{\circ}$ bank, $2000 \mathrm{ft}$, it can be shown that the turn rate will be $40^{\circ} / \mathrm{s}$. Hence, if the pilot were to fly a continuous tight balanced turn, the aircraft's own wake vortex would be met in less than $9 \mathrm{~s}$ - scarcely time for the vortex to have significantly dispersed in even moderately disturbed airflow air. It is known that aircraft flying through the wake vortex of another can suffer a large-magnitude undemanded roll. It is therefore reasonable to assume that the same mechanism as was described above for a loss of visual horizon may also occur, although it is likely that the onset will be more rapid.

The fatal accident to G-MVJU in 1992 was considered by the Air Accident Investigation Branch (AAIB) report to have been a tumble and in-flight break-up following a pilot flying what was observed from the ground to have been extremely tight turns of $360^{\circ}$ or more.

\section{CONCLUSIONS}

This paper has explained that the tumble mode in a weightshift-controlled microlight is sustained by induced flow as an aircraft rotates in pitch about its CG. It has demonstrated that the mode may be initiated by a loss of power in a steeply nose-up pitch attitude, causing the rotation of the trike about the hangpoint to push the wing nose-down, via contact of the front strut and basebar. Using this model, a simple method is shown whereby the conditions of tumble entry can be predicted.

Four possible methods of entry have been explained, through a whip-stall, rolling departure, a failed aerobatic manoeuvre or flight through the aircraft's own wake vortex. All of these occasions are shown to be avoidable through good judgement on the part of the pilot.

The tumble is a potential 'killer' mode in this class of aircraft, as has been demonstrated by history. However, it is shown that, through education and the use of the approach developed in this paper, tumble entry conditions can be predicted and the manoeuvres likely to cause a departure from controlled flight avoided.

\section{ACKNOWLEDGEMENTS}

The authors would like to thank the following for their invaluable assistance in conducting the studies reported in this paper.

Dr Bill Brooks, Technical Director, Cyclone Airsports

Mr Stuart Culling, Senior Engineering Inspector, UK AAIB (now retired)

Mr Mark Dale, Technical Officer, BHPA

Lt Col (Rtd) Chris Finnigan, Chief Executive, BMAA

Mr John Hamer, Test Pilot, BMAA

Mr Roger Pattrick, Chief Designer, Mainair Sports

Mrs Joan Walsh, Engineer and Microlight Pilot

Dr Paul Welsh, Airworthiness Engineer, Flylight Airsports 


\section{REFERENCES}

1 Gratton, G. B. The weightshift-controlled microlight aeroplane. Proc. Instn Mech. Engrs, Part G: J. Aerospace Engineering, 2001, 215(93), 147-154.

2 Gratton, G. B. Less weight more fun. Aerospace Int., February 2000, 30-32.

3 Aviation Safety Review 1990-1999, CAP 701, 1999 (UK Civil Aviation Authority).

4 Brooks, W. G. Report on an accident to a Pegasus Quantum Supersport 503, in Holland, Michigan, USA, on 16 October 2000; http://www.pegasusaviation.co.uk/ pdf/Qtmacc.pdf.

5 Mainair Gemini Flash 2a G-MVEP; Fatal Accident. Bulletin 5/2000: EW/C97/10/5, UK Air Accidents Investigation Branch, 2000.

6 Mainair Gemini Flash 2a G-MTLA; Fatal Accident. Bulletin 1/97: EW/C96/7/8, UK Air Accidents Investigation Branch, 1997.

7 Pegasus XL-Q, G-MVCU; Fatal Accident. Bulletin 8/92: EW/C92/5/1, UK Air Accidents Investigation Branch, 1992.

8 Thomas, J. Report of an incident that occurred on Sunday 21 July at Bedford Microlight Centre (unreferenced report of a recovered in-flight departure submitted to BMAAMainair Gemini Flash 2 G-MNNU).

9 Mainair Gemini Flash 2a G-MTUW, Fatal Accident. Bulletin 9/89: EW/C1 108, UK Air Accidents Investigation Branch, 1989.

10 Mainair Gemini Flash 2a G-MNEJ; Fatal Accident. Bulletin 4/86: EW/C918, UK Air Accidents Investigation Branch, 1986.

11 Mainair Sports Limited, Accident Investigation, Bulletin 20, 1 September 1986 (Mainair Gemini Flash 2 G-MNNF, survived in-flight break-up during flight testing).

12 Mainair Sports Limited, Accident Report, Bulletin 21, 15 September 1986 (Pegasus Flash 2, G-MNYY, survived in- flight break-up during initial testing of a newly built aircraft).

13 Morris, S. J. A simplified analysis of tumbling motion. Hang Gliding, November 1994, 38-44.

14 Fung, Y. C. An Introduction to the Theory of Aeroelasticity, 1955 (John Wiley, Chichester).

15 Bisplinghoff, R. L., Ashley, A. and Halfman, R. L. Aeroelasticity, 1955 (Addison-Wesley, London).

16 British Civil Airworthiness Requirements, Section S, Small Light Aeroplanes, Issue 2, CAP 482 (UK Civil Aviation Authority).

17 Kilkenny, E. A. An experimental study into the longitudinal aerodynamic and static stability characteristics of hang gliders. PhD Thesis, Cranfield Institute of Technology, September 1986.

18 Brooks, W. G. Flight testing of flexwing aircraft; http:// www.raes.org.uk/light-av/brooks_p_1.htm.

19 Microlight Training Syllabus, PPL(A), British Microlight Aircraft Association.

20 Wake Turbulence, General Aviation Safety Sense Leaflet 15A (UK Civil Aviation Authority).

21 Wake Turbulence, AIC 178/1993 (Pink 95) (UK Civil Aviation Authority).

22 Cosgrove, B. Pilots Weather, pp. 45-46 SBN 1-84037-027-0.

\section{APPENDIX}

Time history of a fatal tumble incident, from a failed loop

Note that the origin of this piece of video is not entirely clear. It is believed to have been taken at an airshow in Europe, the aircraft being identifiable as a French 'Cosmos' type. The exact data, location and source cannot be verified. 


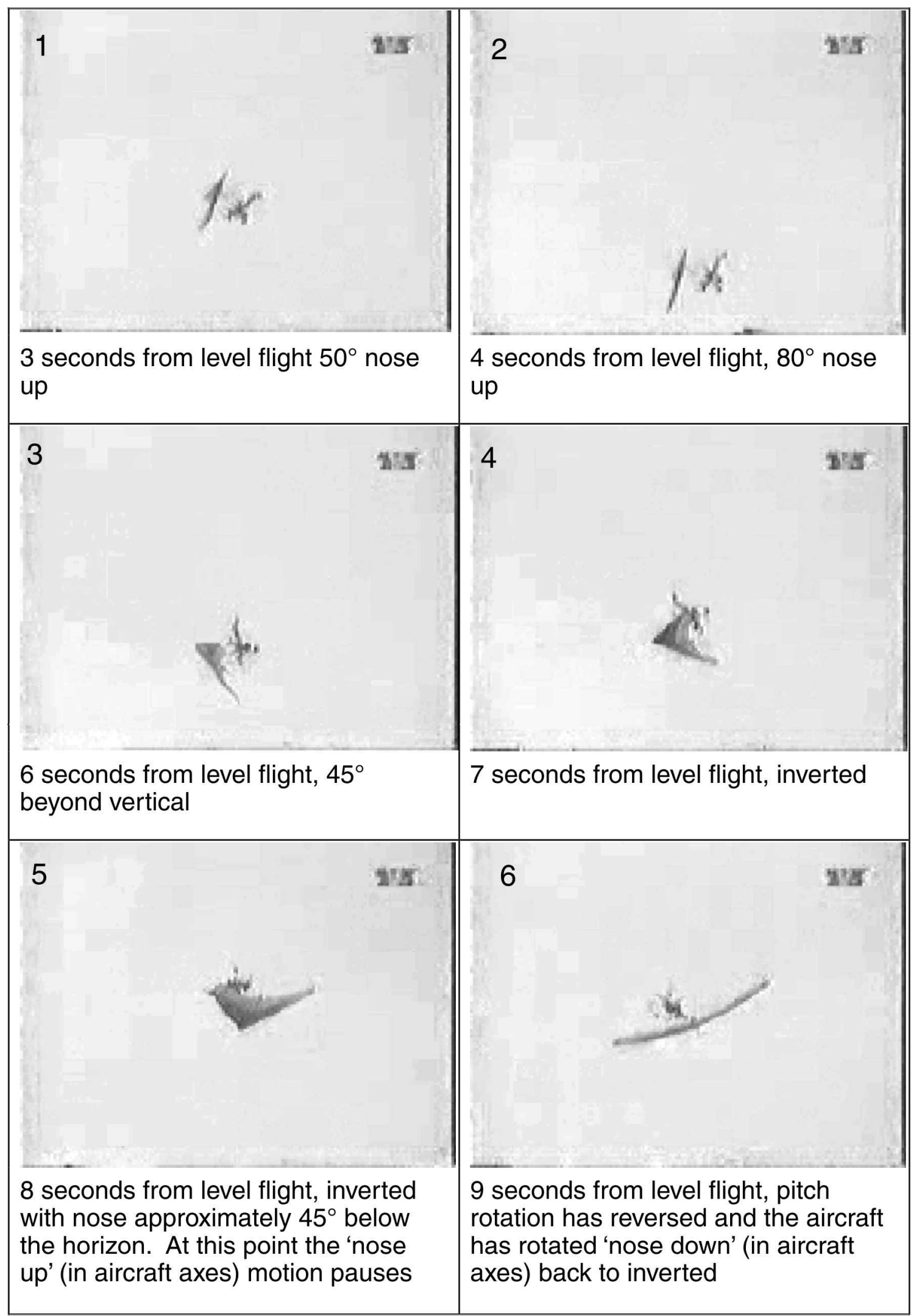

Fig. 18 Video of a fatal tumble incident 


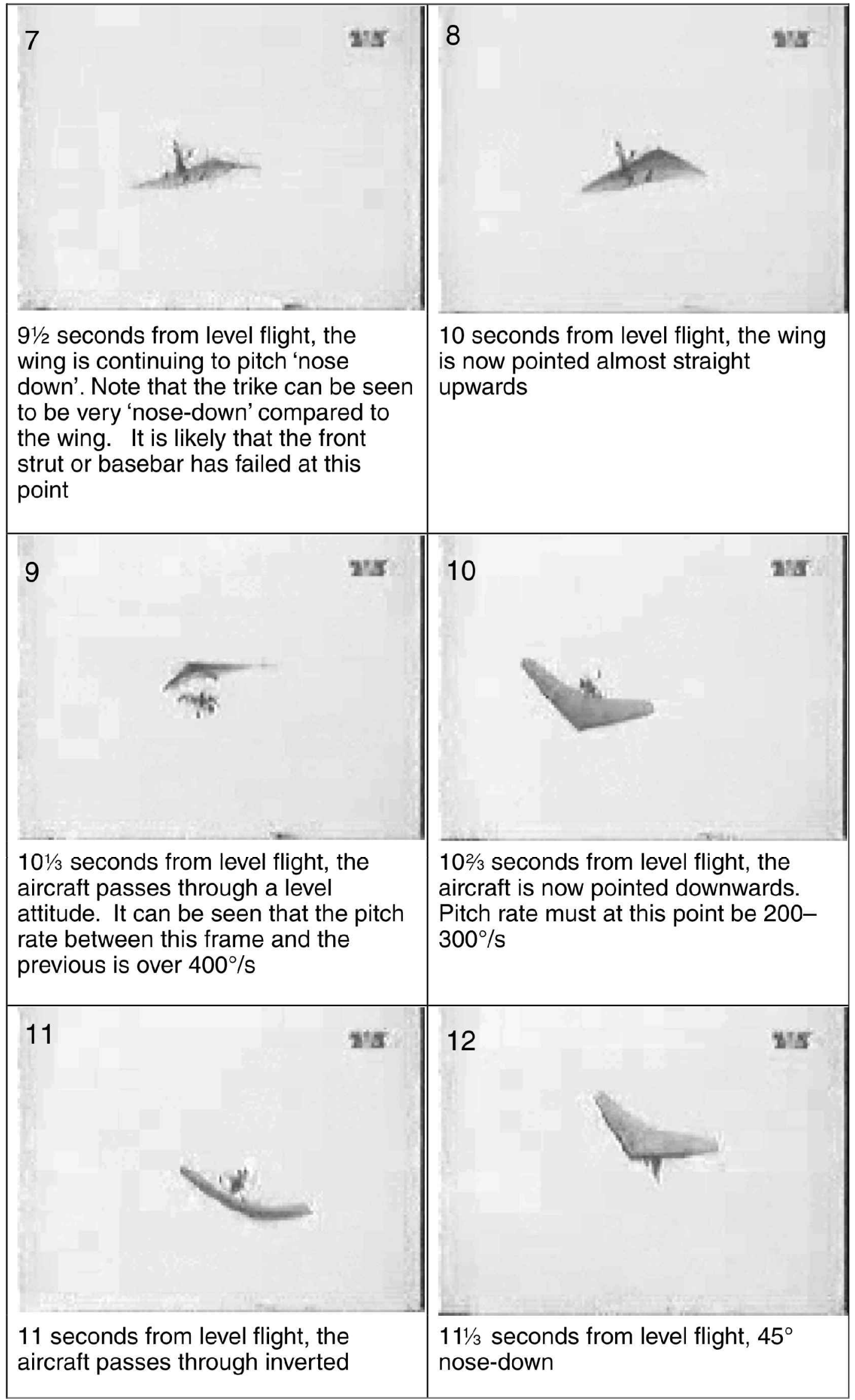

Fig. 18 (continued) 


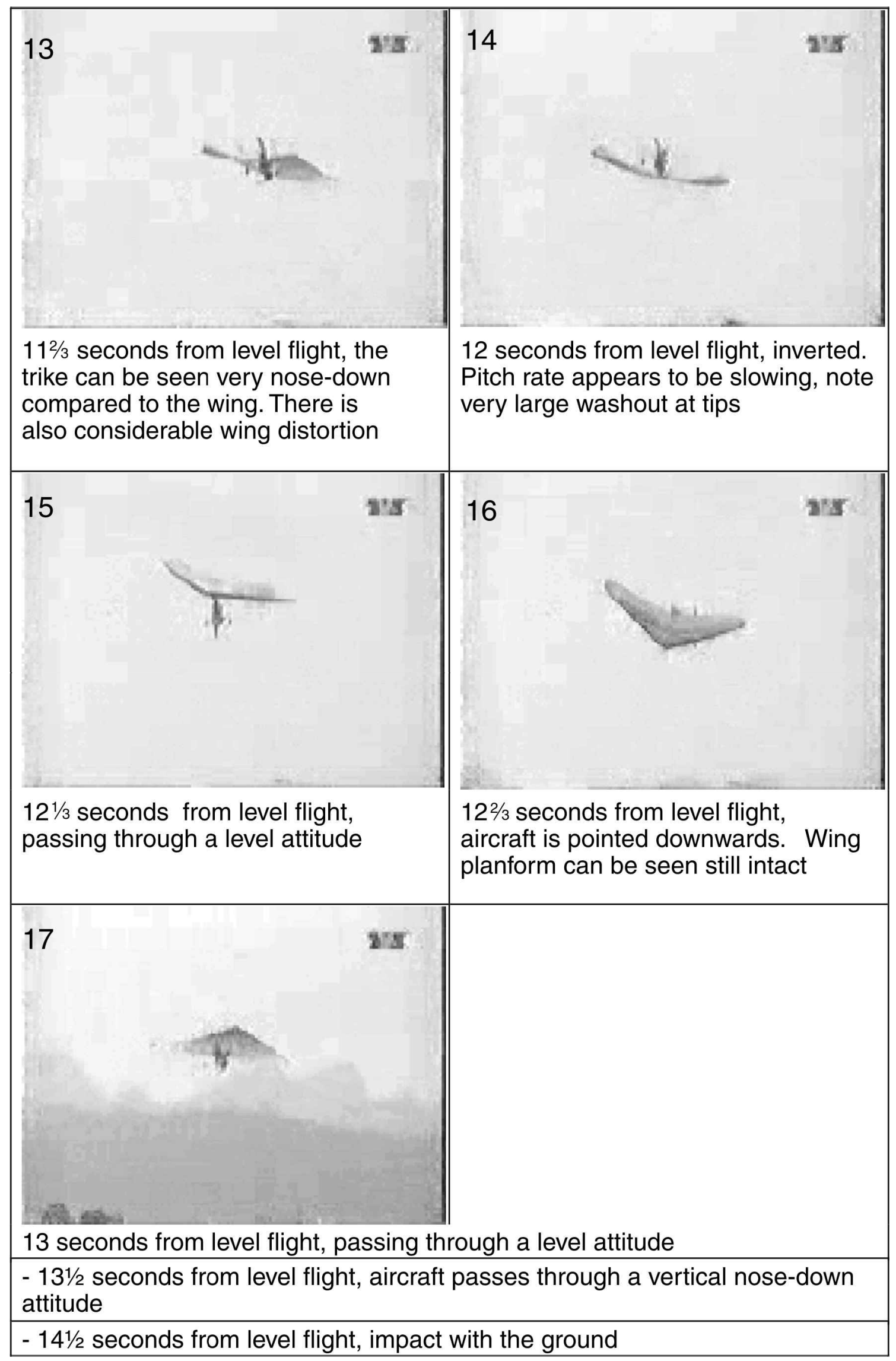

Fig. 18 (continued) 
Copyright $\odot 2003$ EBSCO Publishing 